\title{
El trabajo que no se ve. Dispositivos de inserción sociolaboral para mujeres inmigrantes en Barcelona.
}

\section{The invisible work. Employment and social inclusion devices for immigrant women in Barcelona.}

\author{
Laura Cristina Yufra \\ Investigadora Posdoctoral del CONICET-UNJU \\ laurayufra@gmail.com (ESPAÑA)
}

Recibido: 18.092014

Aceptado: 24.09 .2015

\section{RESUMEN}

El presente trabajo pretende prestar atención a los modos en los que las sociedades de instalación de inmigrantes se dirigen hacia aquellos que son considerados como "otros"; en este caso, las mujeres inmigrantes. Concretamente, nuestra atención se centra en los dispositivos de intervención social que tienen por finalidad facilitar la inserción sociolaboral de estas mujeres. El trabajo de campo, de carácter exploratorio, ha sido llevado a cabo en dos entidades que poseen dispositivos de inserción sociolaboral para mujeres inmigrantes, ambos ubicados en el Barrio del Raval de la ciudad de Barcelona. Las técnicas utilizadas han sido la observación participante y la entrevista. En la descripción de algunos ejemplos de las dinámicas que se producen en dichos dispositivos identificamos cómo se les solicita a las mujeres inmigrantes que realicen ajustes y/o modificaciones de sus modos de actuar para así hacer un uso correcto de los dispositivos de inserción y también para favorecer su inserción sociolaboral. Tales pedidos son analizados a través de las categorías teóricas de Pierre Bourdieu de habitus y violencia o dominación simbólica, revisitadas por algunos de sus críticos. Las mencionadas nociones permiten reflexionar sobre algunas de las interacciones que se producen en dichos dispositivos y sobre la complejidad del proceso de incorporación de las mujeres migrantes a la sociedad de instalación. Del trabajo de campo emerge la orientación de las mujeres inmigrantes hacia los trabajos domésticos y del cuidado, además de cierta desvalorización de la lengua materna de las usuarias y de las manifestaciones visibles de su religión. Aunque se advierte que no son las únicas formas posibles de actuación e interacción, se 
argumenta sobre la importancia de reconocer la existencia de formas jerarquizadas de interacción, con el objetivo de contribuir a una mirada crítica sobre las disciplinas involucradas en la intervención social.

\title{
PALABRAS CLAVE
}

Violencia simbólica - mujeres inmigrantes - inadecuación - dispositivos de inserción sociolaboral.

\begin{abstract}
This paper aims to pay attention to the ways in which immigrant settlement societies deal with those who are considered as «other»; in this case, immigrant women. Specifically, our focus is on employment and social inclusion strategies targeted to facilitate these women's social and work inclusion. Our exploratory field work has been carried out in two social resources which are aimed at work inclusion for immigrant women, both of them located in the Barcelona's Raval district. The techniques used were participant observation and interview. Departing from the description of some examples of interactions established within such entities, we identify how immigrant women are required to do adjustments and / or changes in their behaviour in order to make a proper use of such resources and promote their social and employment insertion. These requirements are critically interpreted in the light of Bourdieu's concepts of habitus and symbolic violence or symbolic domination, reviewed by some critics. These concepts allow us to reflect on the different interactions that occur in these devices. Furthermore, they shed light on the complexity of immigrant women's integration processes into society. The results of empirical research show that immigrant women are orientated to care and domestic work. Other examples confirm a kind of devaluation of the native language of immigrant women and of the visible manifestations of their religion. Even if it is possible to find other forms of interaction in social services; we argue the importance of recognizing these hierarchized forms of interaction in order to facilitate a critical perspective on disciplines involved in social intervention.
\end{abstract}

\section{KEY WORDS}

Symbolic violence - immigrant women - unfitness - settlement support services 


\section{INTRODUCCIÓN}

Los fenómenos migratorios son una de las expresiones de las ingentes desigualdades de la contemporaneidad. A través de las movilidades humanas se conectan diferentes puntos del planeta, en los complejos procesos de emigración e inmigración. Dichas conexiones no son arbitrarias, sino que remiten a historias coloniales, guerras, establecimiento de empresas transnacionales, creación de mercados de trabajo que requieren determinada mano de obra (Sassen 2003, Bade 2000) entre muchos otros factores que podrían mencionarse. Asimismo, cuando se trata de migraciones internacionales, los desplazamientos humanos se encuentran condicionados por las leyes de extranjería de los países y de las regiones supraestatales -como es el caso de la Unión Europea- que limitan y dirigen dichos desplazamientos. La migración femenina, además, pone de manifiesto la persistencia de los patrones heteronormativos de las sociedades de partida e instalación y la división internacional y sexual del trabajo (Phizacklea 1994, Morokvasic 1984). Por otra parte, la crisis mundial de los cuidados (Benería 2008) hace que dicho ámbito del cuidado y del trabajo doméstico se perfile como uno de los sectores laborales de inserción de las mujeres inmigrantes (Anderson 2000, Sassen 2003).

Estos fenómenos tienen una incidencia particular en el contexto español, donde la forma de organización de los recursos del bienestar (o más bien la carencia de algunos de estos recursos) ha influido en la presencia de la migración femenina y en el tipo de inserción laboral de las mujeres inmigrantes (GutiérrezRodríguez 2013).

Como sostiene Gregorio (2010), en España, los estudios sobre género y migraciones en las últimas décadas han formado un "campo" consistente. La autora sintetiza la formación de dicho campo a partir de cuatro grandes preocupaciones temáticas: las maternidades transnacionales, las cadenas mundiales del cuidado, las múltiples discriminaciones que afectan a las mujeres inmigrantes y los cambios que se producen en las relaciones de género a partir de la migración. Ahora bien, reconociendo la multiplicidad de maneras de abordar las migraciones femeninas, las diversas temáticas, dimensiones y niveles que abarca ${ }^{1}$, nos parece de suma relevancia centrarnos en un aspecto que a nuestro entender merece más atención. Esto es, en los modos en los que las sociedades de instalación abordan la presencia (problematizada) de las mujeres inmigrantes a partir de los dispositivos de intervención social que se dirigen a ellas.

El presente trabajo, de carácter exploratorio, se propone presentar y analizar

${ }^{1}$ Entre los numerosos estudios que abarcan este campo en el ámbito español, es posible enumerar, por nombrar sólo algunos, los siguientes: en relación con las familias transnacionales, Parella (2012), Hinojosa Gordonava (2009), Pedone (2007), Suárez Navaz (2007); en referencia a las cadenas mundiales de cuidado: Carrasco (2013), Vega Solís (2009); sobre las múltiples discriminaciones que afectan a las mujeres inmigrantes: Parella (2003), Romero (2003), Colectivo IOE (2001), Escrivá (2000), Catarino y Oso (2000); acerca de los cambios que se producen en las relaciones de género a partir de la migración Pedone (2014), Parella (2012), Gregorio (1998). 
algunas de las dinámicas que se generan en los dispositivos de inserción sociolaboral ofrecidos por organizaciones no gubernamentales. Asimismo, vincularemos dichas dinámicas con los conceptos de habitus y de violencia o dominación simbólica elaborados por Pierre Bourdieu (1991, 1997, 2003) revisadas a la luz de los aportes de Sayad (2010) y Lahire (2004). El trabajo de campo constituye una parte del proceso formativo doctoral de la autora que se vio enriquecido por su participación en proyectos de investigación que abordaron la articulación entre las movilidades humanas, el género y los recursos de atención dirigidos a mujeres inmigrantes en Catalunya ${ }^{2}$.

El artículo se encuentra estructurado de la siguiente manera: luego de una breve contextualización de las formas de organización que han asumido los dispositivos de intervención con inmigrantes en España, repasaremos las coordenadas teórico-analíticas que utilizaremos. Posteriormente, describiremos el trabajo de campo y, finalmente, nos detendremos en el análisis de algunas dinámicas de los dispositivos de inserción dirigidos a la facilitación de la inserción sociolaboral de las mujeres inmigrantes.

\section{DISPOSITIVOS DE INTERVENCIÓN SOCIAL EN EL MARCO DE LAS POLÍTICAS MIGRATORIAS}

Las políticas migratorias de un Estado-nación están constituidas por las políticas de control de flujos migratorios, las políticas de acceso a la nacionalidad y las políticas de integración (López Sala 2005). En este sentido, en el Estado español se han ido instrumentado políticas de integración en los diferentes niveles administrativos. Aunque ha sido cuestionado que sea posible hablar de un "modelo" de integración (Martínez de Lizarrondo 2009), sí se han impulsado planes y programas desde las diferentes comunidades autónomas y gobiernos locales (Zapata 2010) que sirvieron de orientación a las múltiples prácticas de intervención con personas inmigrantes. Estos planes, estrategias y proyectos derivados son influidos por el tipo de Estado de bienestar en los que se ejecutan. El Estado de bienestar español ha sido caracterizado como mediterráneo o familista (Moreno 2002 y Ferrera 1996), por el peso que continúan teniendo las familias en proporcionar bienestar a sus miembros en comparación con el Estado o el mercado (Gil Araújo y González-Fernández 2014), pero también para señalar su menor cobertura en relación con otros Estados de bienestar europeos.

En lo que se refiere a la atención de los inmigrantes, como lo sostienen

2 "Mirando hacia la igualdad. Pertinencia sociocultural de los servicios sociales para mujeres inmigradas ( U-18/06 )" financiado por el Institut Català de les dones y "Mujeres inmigrantes y sistema educativo en la sociedad receptora: Análisis y propuestas de mejoramiento del acceso y la satisfacción en los recursos de formación para la inserción sociolaboral (088/07)" financiado por el Instituto de la mujer. Tales investigaciones fueron llevadas a cabo por el grupo «Fractalidades en Investigación Crítica» de la Universidad Autónoma de Barcelona integrado por Marisela Montenegro, Caterine Galaz, Karla Montenegro y Laura Yufra. 
los planes Pla de ciutadania i immigració (2005-2008 y 2009-2012) y el Plan estratégico de ciudadanía e inmigración (2007-2010) las personas inmigrantes y las mujeres inmigrantes, en particular, son objeto de preocupación por parte de las políticas públicas. En dichos planes se reconoce una desventaja asociada a la condición migrante y al género. De tal modo, las mujeres inmigrantes son identificadas como un colectivo que necesita atención y soporte para la inserción social en igualdad de condiciones, para facilitar su inserción laboral y favorecer la adquisición de habilidades sociales ${ }^{3}$.

Ahora bien, en la línea de lo que decíamos más arriba, tal como sostiene Miguel Laparra (2003) "España ha aplicado a rajatabla el principio de subsidiariedad, otorgando un papel preponderante a las instituciones no gubernamentales de base, en lo que se refiere a la prestación y distribución de servicios y prestaciones sociales específicas para los inmigrantes, mientras que los recursos normalizados y genéricos que recibe el conjunto de la ciudadanía son prestados por funcionarios o empleados públicos." (Laparra 2003: 58). Así pues, el tercer sector no lucrativo se ocupa de las personas inmigrantes, a través del mecanismo de subvenciones y/o acuerdos que se financian con dinero público.

\section{MARCO TEÓRICO-ANALÍTICO}

Para analizar las consecuencias de la intervención social hacia las mujeres inmigrantes desde una perspectiva crítica, encontramos como antecedente los trabajos de Agrela (2004 y 2009) quien reconoce cuatro modelos posibles en la intervención. El modelo paternalista-victimista, que concibe a las mujeres inmigradas como vulnerables, incapaces de hacer frente a las adversidades por sí mismas. El modelo de valoración social negativa, en la que las mujeres migrantes son vistas como anómalas; extrañas que "escapan" fuera del control de sus sociedades de origen, bien sea en calidad de víctimas o de transgresoras. El modelo feminista salvacionista, en el que son percibidas como sujetos apresados por su sociedad patriarcal, mártires de su cultura machista y ancladas en una subordinación que las incapacita para rebelarse. Y el modelo culturalista -que

3 Entre sus líneas prioritarias de actuación en relación con las mujeres inmigrantes se establece el "apoyo a programas integrales dirigidos a mujeres inmigrantes que incluyan elementos tales como: educación de personas adultas, formación profesional, habilidades sociales, inserción laboral y educación para la salud." (Plan de ciudadanía e inmigración 2007-2010: 302). En los planes de la Generalitat de Catalunya se destacan las políticas de primera acogida, que "se anticipen a la desinformación de las personas recién llegadas, que detecten los riesgos a los que se enfrentan y que les ayuden a garantizar un conocimiento correcto de la sociedad de llegada [...] Así como las políticas de igualdad, entre las que se destacan "lo programas destinados a la mejora de la capacidad individual. En este apartado se han desarrollado programas en el ámbito laboral, de las mujeres, de los menores, de la alfabetización básica y del conocimiento de la lengua. Este último programa, 'Impulso y uso del catalán', ha tenido por objetivo facilitar el conocimiento de la lengua catalana a toda la población de Catalunya y, especialmente, a los ciudadanos y ciudadanas de origen extranjero" (Pla de Ciutadania i immigració 2009-2012: 67-68, Traducción nuestra). 
frecuentemente se articula con todos los demás- en el que la cuestión cultural se utiliza como única dimensión explicativa de las necesidades y demandas de las mujeres, en detrimento de otros procesos y factores estructurales que atraviesan la sociedad receptora y condicionan su situación. En nuestro trabajo, no pretendemos reconocer modelos de intervención -aunque sea posible identificar algunos rasgos de dichos modelos- sino identificar algunas dinámicas presentes en los dispositivos de inserción sociolaboral y analizarlos a la luz de algunos conceptos bourdianos ya mencionados.

Martín Pérez $(2009,2010)$, por su parte, ha analizado las interacciones entre inmigrantes y servicios sociales públicos: en las filas para realizar los trámites relacionados con la residencia regularizada de las Oficinas de Extranjería y también en las interacciones que se producen en los servicios sociales públicos (Centros de información y atención de la Seguridad Social, en las Oficinas de empleo y en los Servicios Sociales). En estos trabajos se reconocen diferentes actitudes y representaciones de los funcionarios y de los propios inmigrantes. Dichas diferencias le sirven a Martín Pérez para reconocer, desde las prácticas, el grado de conocimiento y conciencia de los derechos que poseen las personas que solicitan alguno de los servicios mencionados y los diferentes modos de interacción que implican. Así pues se comprende a los servicios públicos como la primera puerta de acceso a los derechos sociales de los inmigrantes, como forma de ejercicio de la ciudadanía social, donde los funcionarios pueden ejercer una "pedagogía" de derechos informando las condiciones de titularidad de los mismos. En el presente trabajo, a diferencia del de Martín Pérez, nos ocupamos de los recursos sociales gestionados por el tercer sector no lucrativo que atiende a personas que no siempre se encuentran en una situación administrativa regular. En este sentido, se advierte sobre los límites de la intervención social antes que sobre el ejercicio de la ciudadanía social.

Como dijimos, nos servimos de las herramientas teórico-analíticas ofrecidas por Bourdieu que permiten reflexionar sobre las solicitudes y demandas que se les realizan a las "usuarias" desde los dispositivos de inserción sociolaboral, así como también sobre las intenciones manifiestas de los mismos -facilitar la inserción sociolaboral de las mujeres inmigrantes- con algunos posibles efectos no deseados que pueden ser comprendidos a la luz del concepto de violencia simbólica.

La definición de habitus como "sistemas de disposiciones duraderas y transferibles, estructuras estructuradas predispuestas para funcionar como estructuras estructurantes, es decir, como principios generadores de prácticas y representaciones que pueden estar objetivamente adaptadas a su fin sin suponer la búsqueda consciente de fines y el dominio expreso de las operaciones necesarias para alcanzarlos, objetivamente 'reguladas' y 'regulares' sin ser el producto de la obediencia a reglas $y$, a la vez que todo esto, colectivamente orquestadas sin ser el producto de la acción organizadora de un director de orquesta" (Bourdieu 1991: 97), logra evitar exitosamente dos riesgos opuestos en el tratamiento de la acción social. Por un lado, la perspectiva del actor plenamente racional calculador de todos sus actos y, por otro, el "actor" 
completamente determinado por la estructura social que condiciona todas sus actividades.

En este sentido, dicha noción remite a la complejidad de los procesos involucrados en los movimientos migratorios, indicando la profunda alteración que supone la migración. Sayad (2010), al analizar los cambios que los emigrados argelinos en Francia deben realizar para llevar a cabo el trabajo industrial-asalariado, sostiene que se trata de un nuevo habitus. De precedente habitus campesino, dichos emigrados se ven obligados a adquirir la mentalidad del cálculo en su inserción al trabajo industrial y, a través de este ejercicio, replicar este comportamiento en el establecimiento de otras relaciones sociales socavando las bases de las antiguas solidaridades familiares ${ }^{4}$. Sabido es que este ejemplo es utilizado en unas coordenadas sociohistóricas muy específicas (el propio Sayad habla de la ejemplaridad de su objeto de estudio), sin embargo, ofrece un antecedente interesante para pensar las transformaciones del habitus en contextos migratorios. El ejemplo migratorio es utilizado, en otro sentido, por Lahire (2004), quien considera necesario reelaborar la noción de habitus. Para este autor es preciso introducir la pluralidad que, a su entender, debe contener dicha noción. El ejemplo de los cambios de habitus que supone la migración, demuestra que las dinámicas de acción responden a un repertorio de esquemas de respuestas posibles antes que a una unicidad coherente de un habitus ${ }^{5}$.Teniendo en cuenta particularmente esta última consideración, es decir, la pluralidad de los esquemas de acción por parte de los actores sociales, así como también que la migración supone modificaciones de las respuestas en tanto que se trata del cambio de un espacio social a otro, las personas que han migrado deberán adquirir nuevos "conocimientos prácticos" que les permiten "estar orientados" en el contexto migratorio. De ello dependerá el poder "jugar" en cualquier situación social gracias a esa precomprensión del sentido del juego, incorporando las estructuras del espacio social en el sistema de disposiciones.

En La dominación masculina (2003) Bourdieu partiendo de su análisis de la sociedad Calibeña -pero haciendo extensible su diagnóstico a sociedades "complejas"- formulará una definición del concepto de violencia simbólica: "La violencia simbólica se instituye a través de la adhesión que el dominado se siente obligado a conceder al dominador (por consiguiente a la dominación) cuando no dispone para imaginarla o para imaginarse a sí mismo o mejor dicho, para imaginar la relación que tiene con él, de otro instrumento de conocimiento

4 "En el aprendizaje de la mentalidad del cálculo que favorece la experiencia del trabajo asalariado y de la vida en Francia, el cálculo de las horas extraordinarias desempeña un papel importante [...] las horas extraordinarias son objeto de una atención minuciosa y perseverante por parte de todos los emigrados, incluso de los analfabetos" (Sayad 2010: 79).

5 "En consecuencia, podríamos proponer la hipótesis de la incorporación por parte de cada actor de una multiplicidad de esquemas de acción (esquemas sensomotrices, esquemas de percepción, evaluación, apreciación, etc.), de hábitos (hábitos de pensamiento, lenguaje, movimiento), que se organizan en tantos repertorios como contextos sociales pertinentes, que dicho actor aprende a distinguir $\neg \neg-\mathrm{y}$ a menudo a designar- a través del conjunto de sus experiencias socializadoras anteriores" (Lahire 2004: 55). 
que aquel que comparte con el dominador, y que, al no ser más que la forma asimilada de la relación de dominación, hacen que esa relación parezca natural; o, en otras palabras, cuando los esquemas que pone en práctica para percibirse y apreciarse, o para percibir y apreciar a los dominadores son el producto de la asimilación de las clasificaciones, de ese modo naturalizadas, de las que su ser social es el producto." (Bourdieu 2003: 51). Por este motivo, la violencia simbólica es un acto de conocimiento de la relación de uno mismo y del otro con el que nos relacionamos. Es, a la vez, un acto de reconocimiento del lugar que ocupan mutuamente dominador y dominado. Pero la violencia simbólica es a la vez una relación de profundo desconocimiento, el dominado no reconoce estas ideas, apreciaciones y acciones como el punto de vista del dominador. La violencia simbólica, se constituye como un sistema de comunicación implícito, aprendido eficazmente a lo largo del tiempo, cuyo carácter de construcción social queda oscurecido para el sujeto. Esto sucede precisamente porque se trata de una somatización, y no un mero proceso de "adoctrinamiento".

Si bien los conceptos bourdianos han sido cuestionados, consideramos que en nuestro abordaje resulta pertinente su recuperación. Las migraciones, como dijimos en la introducción, expresan una direccionalidad, es decir, implican relaciones de desigualdad geopolítica (Sayad 2010) que es preciso reconocer. No obstante, en las interacciones cotidianas puedan darse situaciones de borramientos y desplazamientos que ofrecen otras posibilidades de acción y de reposicionamiento a los actores sociales implicados, por la multiplicidad de esquemas de acción que poseen tales actores. Ahora bien, las respuestas a las solicitudes del nuevo contexto, nunca serán ilimitadas, sino influidas por las relaciones objetivas que se den en un espacio social específico. Así pues, teniendo presentes los matices señalados, será posible captar la complejidad de las interacciones, evitando las posiciones maniqueas, en los efectos indirectos de un conjunto complejo de acciones cruzadas (Bourdieu 2003). De tal modo, será posible pensar tanto las situaciones conflictivas como la no linealidad ni estricta vinculación entre el (implícitamente aceptado) ascenso en la posición social de las personas inmigrantes y su incorporación a la sociedad de instalación.

\section{METODOLOGÍA Y DESCRIPCIÓN DEL TRABAJO DE CAMPO}

El trabajo, de carácter exploratorio, ha sido realizado en dos servicios de inserción sociolaboral dirigidos a mujeres inmigrantes que se encuentran ubicados en el barrio del Raval de la ciudad de Barcelona. Para la realización de este trabajo hemos recurrido al uso de algunas herramientas de la etnografía; la observación participante de las sesiones de orientación e inserción laboral y la entrevista informal. La elección de estas herramientas responde a la necesidad de una descripción detallada de las prácticas cotidianas que se producen dentro de las entidades analizadas y el esfuerzo por recogerlas en su contexto (Hammersley y Atkinson 1994). Asimismo hemos realizado entrevistas semiestruturadas a cinco técnicas referentes de las entidades (a tres técnicas en 
un caso y a dos en otro) y a dos usuarias de cada uno de los recursos. Otros materiales secundarios lo constituyeron las notas periodísticas generadoras de opinión pública sobre la situación de las mujeres inmigrantes así como también los documentos elaborados por las administraciones públicas (Plan Estratégico de Ciudadanía e integración 2007-2010, Pla de ciutadania i immigració 20052008 y 2009-2012).

Como dijimos más arriba, de las personas inmigrantes se han ocupado preferentemente las entidades no lucrativas del tercer sector, fundamentalmente aquellas personas que no han tenido una previa inserción laboral o que se encuentran transitoriamente sin su situación administrativa regularizada. Por estos motivos, hemos decido llevar a cabo nuestro trabajo en este tipo de organizaciones.

La selección de las entidades se realizó a partir del cruce de referencias surgidas: 1) a través de su identificación como entidades destinadas a inmigrantes en la prensa local y por la publicidad de sus actividades; 2) debido a su inclusión en el folleto informativo llamado "Barcelona ciudad de trabajo" -que era entregado en las sesiones orientadoras de la entidad Barcelona Activa dependiente del Gobierno de Catalunya y del Ayuntamiento de Barcelona- y 3) por haber llevado a cabo actividades con una de las universidades públicas de Barcelona.

Las entidades en las que hemos realizado el trabajo se encuentran en el barrio de El Raval, que es el barrio de Barcelona con mayor porcentaje de población inmigrante ${ }^{6}$, asistimos a ellas durante los años 2008 y 2009 de modo intermitente.

Cada uno de los servicios de inserción sociolaboral se imparte en dos instituciones que ofrecen además otros tipos de servicios (acogida, asesoría jurídica, etc.). Una de las entidades (I), destinada a mujeres jóvenes, asume la estrategia de intervención con un programa de un año de duración (aunque la asistencia no es obligatoria) centrada en aspectos de inserción social y laboral. La otra entidad (II), se focaliza en la inserción laboral y pone en marcha estrategias puntuales (asistencia para la elaboración de un currículum; orientación en la búsqueda de recursos para facilitar el empleo; orientación para las entrevistas de trabajo, etc.). Los modos de relación entre técnicas y usuarias son muy heterogéneos ya que la duración de cada una de las estrategias de intervención asumidas establece límites y posibilidades para dichas relaciones. En el primero de los casos, se trata de un conocimiento personalizado de cada una de las asistentes por parte de las técnicas (son once las usuarias que asisten regularmente al dispositivo durante el periodo en el que transcurre la investigación). En el segundo, la relación es más distante debido a la corta duración de la intervención y fueron diecinueve las mujeres inmigrantes que acudieron a este recurso en los momentos de observación participante. Las

${ }^{6}$ El barrio del Raval es el barrio que cuenta con la tasa más alta de inmigración de la ciudad (110,9 cada mil habitantes). Departament d'Estadística, Ajuntament de Barcelona (2012). Disponible en http://www.bcn.cat/estadistica/catala/dades/barris/tdemo/imi/taxes2012.htm 
entrevistas informales llevadas a cabo con las usuarias fueron realizadas en los espacios de recreación, en el momento de llegada o de finalización de las actividades.

La entidad (I) trabaja también en otros ámbitos de intervención social (infancia, familia, etc.). La entidad (II) posee una clara adscripción al mundo árabe. Ambos servicios cuentan con un trabajo ampliamente reconocido por los vecinos del barrio.

\section{RESULTADOS}

\subsection{Favoreciendo ¿la integración?}

Los dispositivos de intervención social se proponen, mediante su accionar, reparar o equilibrar las situaciones de desventaja de las usuarias y conseguir que la utilización del servicio sea pasajera, hasta que ellas adquieran las habilidades para no necesitar más de él ${ }^{7}$. Entre los servicios que se imparten podemos nombrar la enseñanza de las lenguas de la sociedad de instalación (castellano y catalán), el manejo de las nuevas tecnologías, orientación sobre las oportunidades que ofrece la ciudad, la confección del Curriculum Vitae etc. Esta formación será planificada pero también espontánea (no planificada) y apuntará a la adquisición de aquellos saberes prácticos que difícilmente se tematizan de modo explícito. Una clave de interpretación de las relaciones que se producen en los dispositivos de intervención apunta a que la tarea que realizan dichos dispositivos consiste en la facilitación de la adquisición de nuevos habitus, esquemas de acción, por parte las mujeres inmigrantes obteniendo el conocimiento práctico necesario para orientarse en el nuevo contexto social.

Decidimos centrarnos allí donde se veía una inadecuación o inadaptación de las usuarias a la nueva situación social para encontrar claves de lectura en la interpretación de las interacciones entre técnicas y usuarias. Así pues, nos detuvimos en el tipo de acciones o las respuestas de las usuarias consideradas inadecuadas por las técnicas. En esta particular interacción, se ponen de manifiesto las expectativas hacia las usuarias de su forma de comportamiento en un determinado contexto.

El primer aspecto que se pone de manifiesto es la distinción entre las formas adecuadas de comportamiento en el propio dispositivo de intervención y lo que sucede fuera de dicho dispositivo. Dicha distinción introduce el accionar de los dispositivos de intervención en un contexto de actuación más amplio.

\section{a) Saber jugar en los dispositivos de intervención:}

7 En el cuaderno de campo se registra la definición que una técnica hace de la "autonomía" de las usuarias: el objetivo del servicio es la autonomía, [...] Por ejemplo ella a veces les lee los anuncios, y claro, no lo podrá hacer siempre, ya se tendrán que 'espabilar' Entidad II entrevista informal con técnica de inserción sociolaboral, contratada por la entidad, de origen catalán con estudios universitarios. 
Hablando sobre la importancia para la integración de las usuarias del conocimiento del idioma, N., técnica de acogida de origen marroquí, destaca lo que va más allá del puro conocimiento de la lengua y tiene que ver con ese conocimiento práctico que permite actuar adecuadamente en una situación, en este caso hacer un uso provechoso de los servicios,

[...] a mi me pasa que hay personas que hablan en mi propio idioma y no me quieren entender y si no me quieren entender pues por mucho que hable con ellas no me van a entender porque ellas quieren que se les dé la solución que ellas quieren si tú no la das, es como que no te entienden [...] Entidad II, entrevista con N., técnica de acogida, contratada por la entidad, marroquí, con estudios universitarios completos en el país de origen

La precedente cita, expresa cómo $\mathrm{N}$. interpreta el comportamiento de algunas de las usuarias de la entidad como una incapacidad de éstas al no poder poner en relación su demanda con las posibilidades concretas del servicio. Desde este punto de vista, se trataría de una actitud inadecuada solicitar algo que se encuentra más allá de las posibilidades de la entidad. Las expectativas de las usuarias, según N., deberán ajustarse a los recursos con los que cuenta la entidad. Tal es el comportamiento esperado: a partir de la interacción con el dispositivo de inserción sociolaboral, las usuarias paulatinamente modificarán su comportamiento para que la intervención pueda resultar provechosa ${ }^{8}$.

Otra consideración a destacar es que en el marco del dispositivo de intervención, las técnicas de las entidades se encuentran en una relación de poder a través de los conocimientos y las herramientas que poseen para facilitar la inserción sociolaboral que las usuarias anhelan. Como el espacio es relacional, las usuarias en comparación con las técnicas ocupan una posición de no-saber sobre aquello que ofrecen las entidades (la enseñanza de la lengua, la orientación al medio de acogida, cómo presentarse en una entrevista de trabajo, los saberes informáticos etc.). Por lo tanto, las interacciones sociales se dan entre algunas que "saben" y tienen que trasmitir dicho saber y otras que "no saben" y buscan obtener dichos conocimientos. Son las técnicas las que desarrollan las sesiones en las que se trasmitirán contenidos que las usuarias deberán aprender. Ahora bien, cada uno de los agentes sociales se pone en juego y, por esta razón, las posiciones no son fijas ni inmutables. Un ejemplo de ello lo pudimos reconocer

8 Martín Pérez (2010) reconoce como una categoría de usuarios que asumen una actitud "conflictiva" a quienes no saben identificar cuáles son los derechos de los que son portadores. "La «queja» se refleja en interacciones claramente conflictivas en las que se muestra una visible hostilidad hacia los funcionarios y hacia toda la organización administrativa [...] Se reclama un derecho que no existe, que no está reconocido o al que, por determinadas razones, no se tiene acceso [...] Este tipo ideal reflejaría una cierta concepción «reivindicativa» de la ciudadanía, aunque el alcance de dicha reivindicación, en el ámbito de las oficinas públicas, únicamente se pueda reflejar como una queja interpretada negativamente: a estos individuos se les identifica con personas que acuden a las oficinas públicas con el único fin de influir negativamente en el «buen funcionamiento» del servicio público" (Martín Pérez 2010: 68). 
en una situación de observación:

J. [la técnica] le dice a F. que se calle y advierte que tiene un chicle, le dice que lo tire. Continua dirigiéndose a H., mira a F. y ésta le dice que ha tirado el chicle, y abre la boca y le dice: "mira". J. continúa hablando y ve que F. aún tiene el chicle. Se enfada y dice "esto es muy fuerte”. J. dice que se acabó la clase. Entidad I, observación participante, sesión de orientación para el empleo

A pesar de que las usuarias se encuentran en la posición de no-saber en relación con los conocimientos específicos del servicio, esto no quiere decir que acepten pasivamente todas sus demandas y exigencias. Cierto es que por el episodio relatado la sesión fue interrumpida, sin embargo, se pone de manifiesto cómo las posiciones sociales dentro de un determinado contexto están puestas en disputa y se trata de un proceso dinámico. No obstante, una vez más, la actitud de F. no es considerada adecuada desde el punto de vista de la entidad y por ello requiere una intervención correctora.

Otro ejemplo en el que se manifiesta un desajuste de las expectativas del comportamiento de las usuarias en relación con el dispositivo de intervención se refiere al tema de la puntualidad. Durante el trabajo de campo se observaron numerosas situaciones en las que las técnicas intervienen sobre este aspecto que consideran una forma de comportamiento inapropiado. Se produce la siguiente situación cuando L., una usuaria, llega quince minutos tarde a una clase de orientación laboral. Sus compañeras estaban realizando la simulación de una entrevista de trabajo.

L. dice con mucho entusiasmo que quiere participar. $Y$ dice algo así como “¿qué es?, ¿qué pasa?, ino entiendo!” a lo que J. responde "si hubieses llegado a tiempo, lo sabrias”. Entidad I, observación participante sesión de orientación laboral.

La exigencia de puntualidad por parte de J. llama la atención particularmente por el hecho de que ni ella ni la profesora de catalán eran puntuales ${ }^{9}$.

En la entrevista realizada con M., coordinadora del dispositivo de intervención, se trae a colación una discusión sobre los horarios,

[...] lo que haces aquí es un poco lo que te vas a encontrar mañana o sea, sé puntual, porque el día de mañana en una empresa tendrás que ser puntual, eso es una cosa de cada día, a las nueve aquí, no me hables en árabe porque cuando estés en una tienda no podrás hablar en árabe, ven bien arreglada, dúchate, son pequeñas cosas, son pequeños hábitos pero que nosotros ya los tenemos más que asumidos, quizás, pero a ellas les cuesta. Una buena manera trabajando así, cada día a las nueve '...Ah...pero es que yo me levanto a las diez'...me es igual... [...].

${ }^{9}$ La profesora de catalán era una voluntaria de la entidad. Esto lleva a reflexionar sobre las condiciones en las que se desarrollan muchas de las intervenciones sociales en el tercer sector no lucrativo. 
Entidad I, entrevista M., coordinadora del dispositivo de intervención, catalana, con estudios universitarios completos

Algo similar sucede con el pedido de las técnicas (de la Entidad I, M. coordinadora del dispositivo de intervención y J., técnica de inserción laboral; de la Entidad II, T., técnica de inserción laboral y N., de acogida) de que las usuarias informen sobre inasistencias o tardanzas a través de llamadas telefónicas o mensajes de texto. En este caso, también se apela al futuro rol de trabajadoras para insistir en la necesidad de poner sobre aviso a sus posibles empleadores ante alguna situación como las mencionadas.

Así pues, en los dos ejemplos precedentes se introduce un elemento de exterioridad al dispositivo de intervención. Se pone en relación las reglas de funcionamiento de la institución con aquello que está más allá del dispositivo la sociedad, el mercado laboral, etc.

\section{b) Saber jugar más allá de los dispositivos de intervención:}

Los ejemplos precedentes, como dijimos, ponen de manifiesto la norma de la "puntualidad" que el dispositivo "enseña". La justificación plena de su importancia se encuentra en las reglas del mercado laboral. Es decir, las usuarias deberán incorporar ese saber práctico, la puntualidad, requisito indispensable en el mercado laboral. Ahora bien, esta "demanda" no es cuestionada por los dispositivos de intervención sino que se asume tal lógica de funcionamiento como propia.

Todo lo contrario sucede con el ejemplo que sigue a continuación. Durante la entrevista con E., técnica de inserción laboral, reconoce que el uso del pañuelo de las mujeres musulmanas es considerado como un elemento que constituye un impedimento para el acceso al trabajo.

[...] muchas de ellas van con pañuelo, ¿vale? son musulmanas y con pañuelo y, a la hora de buscar trabajo, el pañuelo les es un impedimento, es una realidad, es así y lo ves. [...]” Entidad II Entrevista con E., técnica de inserción laboral, contratada por la entidad, catalana, con estudios universitarios completos.

Esta exigencia del mercado laboral, que es reconocida por la técnica como arbitraria, sin embargo, se presenta en su relato como una regla que debe ser conocida para una mayor eficacia en la búsqueda y obtención del empleo ${ }^{10}$. Forma parte de las funciones de los servicios que sus "usuarias" reconozcan

10 Las investigaciones de Bauder (2005) sobre inmigrantes provenientes del sur de Asia y de la ex Yugoslavia en Vancouver señala, en consonancia con lo dicho, cómo el mercado laboral predefine los comportamientos esperados de las personas inmigrantes: "Las convenciones y normas del mercado laboral canadiense semejan un conjunto de 'reglas' que orientan las expectativas de comportamiento. [...] Los inmigrantes que no son capaces de seguir adecuadamente las reglas del mercado laboral canadiense no son competitivos en comparación con los trabajadores canadienses quienes conocen y obedecen las reglas" (Bauder 2005: 3). 
estas reglas del mercado laboral, sin embargo la entidad no la asume como regla de funcionamiento propia.

Todas estas sutiles formas de saber práctico son las que pueden definir si una persona es empleable, a la vez que pueden expresar su capacidad de dominar mejor las reglas del juego no ya para el funcionamiento interno del dispositivo, sino fuera de él, en el mercado laboral.

Las siguientes consideraciones iluminan este aspecto. La entidad I organizó un curso de formación laboral de monitora de comedores escolares, J., la técnica de inserción laboral relató el desempeño de A.,

[...] yo tengo una de las chicas que hizo las prácticas y durante tres meses no se quedó a comer ni un día con el equipo de monitores y no hablaba con nadie. Para el coordinador, obviamente, me dijo que esta chica no...no está preparada, pero no porque no pueda y bueno, más o menos, iba haciendo, era como una hormiguita, iba haciendo, pero no decía nada, si haces de monitor, tienes que moverte un poco, ahora hablas con uno, pero ella no se quedaba a comer para no hablar ¿sabes? [...] yo flipé es A., ¿sabes A.?, es ella, que no hablaba. Se cohibía y no podía. Entidad I, entrevista con J., técnica de inserción sociolaboral contratada por la entidad, catalana, con estudios universitarios completos

El ejemplo revela cómo la "incapacidad" de A. no consistía en una falta de conocimientos, sino en la carencia de aquellos otros esquemas de acción que le permiten poder desenvolverse en las diversas situaciones sociales relacionadas con la tarea concreta que la usuaria pretendía desempeñar.

En la misma línea de argumentación, describimos a continuación otro ejemplo que apunta a la necesidad de trasmitir un "conocimiento práctico" que permitirá desempeñarse a K. según las expectativas. La siguiente situación se da en la Entidad II cuando T., técnica de inserción laboral, está apuntando a K., usuaria de origen marroquí de 45 años, parada, en una oferta de trabajo publicada en un portal web,

Hay una pregunta sobre las expectativas del sueldo. T. se la explica a K. y ella responde ‘¿tengo que responder sin mentir cuánto quiero ganar?’ La técnica le matiza el 'sin mentir'. K. dice l200€ y T. se lo pone un poco justificándolo, 'teniendo en cuenta que trabajaría los fines de semana....'. Entidad II, observación participante, sesión de inserción laboral.

En la mencionada inscripción, T. justifica la respuesta de K. ya que considera que su respuesta no es del todo acorde con la oferta laboral, intentando de este modo facilitar las posibilidades de contratación de N. en un mercado laboral muy competitivo. Para que K. pueda conseguir trabajo deberá aspirar a un sueldo más bajo o bien justificar por qué merecería tener un estipendio más alto. Como esto no lo "sabe" K. (lo intuye, quizás, con la pregunta acerca del "sin mentir"), la función que lleva a cabo T. es asumir el punto de vista del mercado laboral y hacérselo conocer.

En las descripciones que acabamos de hacer nos hemos centrado en las 
“inadecuaciones” que, según los dispositivos de actuación, las usuarias deberán modificar para lograr una mejor inserción sociolaboral. Sin embargo, no siempre se trata de marcar las inadecuaciones, también se reconoce la capacidad de adaptación de las mujeres inmigrantes al nuevo contexto. Así lo resume N.,

[...] una persona que migra ya es muy adaptable si no, no lo haría, es decir que yo creo que son muy fáciles de adaptar, además tienen una mente muy abierta, intentan aprender, si por ejemplo en el caso del servicio doméstico, pues seguramente en el caso de las marroquíes, por ejemplo, en su país limpian de otra manera, o sea, es diferente pero aprenden muy rápido [...].Entidad II, entrevista con N., técnica de acogida, contratada por la entidad, marroquí, con estudios universitarios completos en el país de origen.

Como vemos, no sólo se destacan las inadecuaciones de las usuarias sino también sus formas pertinentes y adecuadas interacción. Lo que queda claro, no obstante, es que la migración exige un proceso de modificaciones de las formas de hacer habituales de las mujeres inmigradas.

Un último aspecto que queremos señalar es, como señala M., la coordinadora del dispositivo de intervención de la Entidad I, son los intentos de pactar con empresas para conseguir trabajos para sus usuarias pero que las empresas "no se quieren mojar". Sostiene que las empresas afirman que desean colaborar con la institución pero que, llegado el momento de hacerlo, no se comprometen. En un caso en el que estuvieron a punto de concretar un acuerdo de inserción laboral en la que la entidad asumía la formación requerida por la empresa, ésta finalmente no quiso asegurar la contratación de las usuarias que hubieran finalizado exitosamente la formación. En otro tramo de la entrevista, en relación con la contratación de las personas que no tienen su situación regularizada señala:

[...] tienes que hacer una oferta de trabajo convencer al empresario que les haga una oferta de trabajo y yo creo que ahi es dificil, porque "eso es muy complicado" y no le es, porque es sólo traer unos cuantos documentos e ir al gobierno civil. Pues ya, como hay poca información para los empresarios, es ya un trabajo, una molestia, ya es el más grande de los problemas. Entidad I, entrevista M., coordinadora del dispositivo de intervención, catalana, con estudios universitarios completos.

Las demandas "exteriores", en algunos casos, se asumen para el funcionamiento del dispositivo de intervención como por ejemplo, la puntualidad o el avisar en caso de algún impedimento para la asistencia. En cambio, en otros, no asume la misma perspectiva, por ejemplo, la demanda de evitar el uso del pañuelo por parte de las mujeres marroquíes. A pesar de esta última opción, los requerimientos del mercado laboral para lograr la inserción laboral de sus usuarias se presentan como difícilmente modificables por parte de los dispositivos. 


\section{2. ¿Violencias simbólicas?}

Como venimos argumentando, desde los dispositivos de intervención se señalan "inadecuaciones" por parte de las mujeres inmigrantes, solicitándose cambios y modificaciones de las conductas y comportamientos de sus "usuarias". Lo que se comprenda como inadecuación, responde a motivos sumamente complejos $^{11}$ que sólo hemos podido abordar aquí desde los emergentes del trabajo de campo.

\section{a) Perfiles laborales}

El trabajo doméstico y de los cuidados ha sido y continúa siendo la principal vía de acceso al trabajo para las mujeres inmigrantes no comunitarias (Gil Araújo y González-Fernández 2014, Gutiérrez-Rodríguez 2013, Colectivo IOE 2001).

Desde los dispositivos de inserción sociolaboral también se apunta a tales sectores como ámbito de efectiva inserción de las mujeres inmigradas. En entrevista con E., técnica de inserción laboral de la Entidad II, reconoce las múltiples capacidades de las mujeres inmigrantes, que se ven abocadas a las tareas del cuidado.

No, a ver, estamos hablando de que la mayoría de personas inmigrantes, en el caso de las mujeres inmigrantes trabajan en servicio doméstico, cuidado de personas mayores, restaurantes y hay muchísimas mujeres capaces, que tienen licenciaturas, son diplomadas o tienen capacidades ya no formativas sino de ellas mismas que pueden hacer, ejercer otros trabajos que no se les ofrece, pero como dicen aquí, aquí no se los dan ni a las propias autóctonas, claro, es muy complicado, viniendo de otro país con una formación que puede, a veces, aportar mucho más. Entidad II Entrevista con E., técnica de inserción laboral, contratada por la entidad, catalana, con estudios universitarios completos.

La existencia de los perfiles laborales tan marcados para las mujeres inmigrantes y el reconocimiento de la dificultad para modificar dicha situación puede producir la "adaptación natural" de las mujeres inmigrantes a este tipo de trabajos de cuidados, a pesar de poseer otras calificaciones profesionales ${ }^{12}$. En este sentido, los dispositivos de inserción también orientan a la inserción laboral en estos perfiles laborales, ya que son identificados como ámbitos donde es posible

11 Por ejemplo, basadas en "la interpretación miserabilista que se realiza de los países de los que provienen las personas migrantes 'no comunitarias' como 'atrasados', 'subdesarrollados', que, no habiendo podido acceder todavía a la modernidad, siguen inmersos y bloqueados en las cada vez más fangosas arenas de la tradición o de la premodernidad" (Santamaría, 2002: 130). Asimismo, al tratarse del ámbito de inserción laboral, las inadecuaciones señalas remiten, como hemos señalado, a todas las formas de disciplinamiento en el trabajo.

12 El trabajo de Ribas et al. (2000) sobre servicios de formación dirigidos a mujeres inmigrantes, concluye que existe "filosofía asistencialista y asimilacionista, de modo que no aprovechan los conocimientos, los hábitos y las aptitudes que estas mujeres adquirieron en sus sociedades de origen" (Ribas et al. 2000: 10). 
la inserción laboral más inmediata, consolidando una especie de "destino" de inserción laboral. Por lo dicho, podemos suponer que si una mujer inmigrante aspira a cobrar un sueldo considerado alto o evitar las tareas domésticas y del cuidado, le resultará mucho más difícil su incorporación efectiva al trabajo que si aspira a los perfiles laborales identificados trabajos de "mujeres inmigrantes". Y esto sucede a pesar de contar con competencias diversas, tal como se señala en la entrevista. Ahora bien, el mismo fragmento de entrevista revela formas de reconocimiento de las competencias que poseen las mujeres inmigrantes aunque, a la vez, patentiza la dificultad de hacerlas valer en un mercado laboral generizado y competitivo, donde el trabajo de cuidados y doméstico, forma parte del trabajo precario con escasa valoración social.

\section{b) Devaluación de la lengua materna en los dispositivos de intervención}

En el siguiente ejemplo, se deja entrever la escasa valoración de la lengua materna que las propias usuarias realizan en el contexto de los dispositivos de intervención. En un ejercicio de orientación laboral en el que se habían explicado la definición de competencias y habilidades, las usuarias debían señalar qué competencias y habilidades pedirían ellas a una canguro para cuidar a sus hijos, las respuestas que cada una había pensado y escrito fueron puestas en común en la pizarra.

Las ideas que surgen son, por ejemplo, saber si el niño está enfermo, que sea responsable, puntual, que sepa limpiar al niño, qué sepa divertir al niño, que sepa castellano y catalán. Sólo B. dice que sepa árabe. El resto de las chicas (K., L., A., H. y S.), dicen que pedirían a una canguro el conocimiento del catalán y el castellano. Entidad I, observación participante, sesión de orientación laboral.

La lengua propia tiene valor social secundario y esto impide reconocerlo como conocimiento pertinente y necesario en el marco del dispositivo de intervención para la mayoría de las usuarias. El ejercicio suponía la expresión espontánea de ideas, es decir, la interiorización de un punto de vista. En dicho ejercicio se puso de manifiesto la importancia de las dos lenguas del país de instalación para el eventual cuidado de sus propios niños (hijos de madres arabo-parlantes). Esta prevalencia de las lenguas del contexto de inmigración deja traslucir cómo en los dispositivos de intervención se oscurece la valía del propio conocimiento lingüístico de las usuarias.

Con esto no queremos decir que las usuarias no utilicen cotidianamente su lengua materna en los dispositivos de inserción, cuyo contenido comunicativo excluye de su comprensión a las personas no arabo-parlantes. Sin embargo, el ejemplo ilustrado advierte sobre las formas de valoración social del árabe en la sociedad mayoritaria.

\section{c) Formación recibida con diferente validez}


La formación impartida en el marco del dispositivo de intervención no tendrá la misma validez formal para las usuarias que no posean el permiso de trabajo. La formación impartida, requerida para obtener el Certificado de Monitor de ocio infantil y juvenil, no tiene la misma validez formal para las usuarias que se encuentran en la situación mencionada. Tampoco pueden acceder a las certificaciones de catalán emitidas por Consorci per a la Normalització Llingüística $^{13}$, a pesar de que la entidad ofrece formación de dicho idioma. En la entrevista con M., advierte sobre la importancia de que las usuarias sepan de las limitaciones del tipo de permiso que poseen en relación con la formación ofrecida,

[.... tú tienes un tipo de residencia que no te permite trabajar. Esto también tiene que estar muy claro, o 'si haces prácticas, pues este año no te van a coger,' sabes, también explicarles las cosas muy bien y que lo tengan muy claro [...] Entidad I, entrevista M., coordinadora del dispositivo de intervención, catalana, con estudios universitarios completos.

La formación que las usuarias realizan no tiene reconocimiento oficial si no poseen un permiso de residencia que las habilite a hacerla. Ahora bien, la certificación académica expresa la adquisición de competencias en una determinada materia, pero en el caso de las usuarias de los dispositivos, a pesar de haber completado su formación, no obtendrán el reconocimiento oficial de sus estudios.

La orientación de las usuarias a las demandas del mercado laboral más comunes (limpieza y cuidado de las personas) como un "destino" más allá de sus disposiciones, saberes, habilidades y capacidades se ve reforzada en el tipo de formación y las herramientas que son ofrecidas por las entidades que van orientadas a este tipo de trabajos. También es preciso atender a aquellos efectos de desvalorización de la lengua materna, las manifestaciones exteriores de la religión musulmana o la valencia desigual de las certificaciones de los procesos formativos por la falta de reconocimiento oficial. Así pues, perviven formas más o menos sutiles de jerarquías en función del origen nacional o étnico y en función del género, recreándose posiciones de superioridad e inferioridad entre las personas que interactúan, que pueden ser revertidas de múltiples maneras, aunque también reafirmadas

\section{CONCLUSIONES}

Con la expresión "el trabajo que no se ve" nos referimos a la acción cotidiana de los recursos de inserción laboral para mujeres inmigrantes que trasmiten pautas, valores y posiciones jerarquizadas entre las personas que

${ }^{13}$ Entidad dependiente de la Generalitat de Catalunya que se ocupa de facilitar el conocimiento, el uso y la divulgación del catalán en todos los ámbitos. Los certificados emitidos se encuentran homologados con los niveles reconocidos para las otras lenguas oficiales de la Unión Europea. 
interactúan en ellos. Pero también remite a los procesos invisibilizados de incorporación de relaciones jerarquizadas por parte de las mujeres inmigrantes en determinados contextos de la sociedad de instalación. Asimismo la expresión remite a las tareas domésticas y de cuidado, ámbito privilegiado de ocupación de las mujeres inmigrantes que, pareciera invisible y de escaso valor social, aunque la propia reproducción de la vida dependa de él (Carrasco 2013).

El carácter acotado del trabajo no pretende ofrecer una mirada simplificadora de las dinámicas que se producen en los recursos de inserción sociolaboral para mujeres inmigrantes y de las solicitudes que desde allí se les solicitan como expresión de las demandas de la sociedad de instalación. Para ello, sería necesario un trabajo mucho más amplio y sistemático. Sin embargo, el presente análisis consideramos que ofrece algunas luces sobre algunas características que pueden estar presentes y sobre las que es preciso reflexionar.

Hemos reconocido cómo desde los recursos de inserción sociolaboral se señala la presencia de una "lógica exterior" que a veces se asume como lógica de funcionamiento propia, aunque, en ocasiones, eso no sucede. Sin embargo, dicha "lógica exterior" en cualquiera de los dos casos se presenta como difícilmente modificable desde los propios dispositivos de intervención. En la medida en que los dispositivos no ofrecen herramientas para imaginar una forma de reconocimiento y de establecimiento de relaciones diferenciadas a las demandas de estas lógicas mayoritarias que clasifican a la inmigración como "problema social" es preciso llamar la atención sobre dinámicas que pueden convertirse en formas de violencia simbólica. En este sentido, el presente trabajo constituye una apuesta para que las disciplinas comprometidas en la intervención social, apunten como prioridad la mirada crítico-reflexiva sobre las propias prácticas.

Como ya lo mencionamos, con los ejemplos ofrecidos no pretendemos negar la posibilidad de otros recorridos e interacciones en el ámbito de la intervención. Sabido es que existen múltiples trayectorias de inserción entre las mujeres inmigrantes así como también en otros ámbitos sociales cotidianos (lúdicos, deportivos, de participación política, etc.) con lógicas diferentes a las mencionadas. Así también, en relación con el idioma, además del ejemplo reseñado más arriba, el árabe es uno de los idiomas de comunicación cotidiana en los dispositivos de inserción sociolaboral. Lo mismo sucede con las sugerencias en relación con las manifestaciones de la religiosidad musulmana, de hecho, el uso del pañuelo es un signo visible en los espacios públicos y al interior de los dispositivos de intervención. Esto es así ya que las interacciones deben ser comprendidas en sus complejos contextos. Conocer dichos contextos permite identificar entramados sinuosos de inclusiones y exclusiones, de ordenaciones y subordinaciones y asimismo establecer puentes de articulación, como condición para la construcción de políticas de solidaridad (Brah 1987). Consideramos, pues, para finalizar, que tales políticas de solidaridad constituyen un desafío que merece ser tenido en cuenta desde un punto de vista académico, institucional y político. 


\section{BIBLIOGRAFÍA}

AGRELA, B. (2004): "La acción social y las mujeres inmigrantes: ¿hacia unos modelos de intervención?", Portularia, 4, pp. 31-42.

AGRELA, B. (2009): "De los significados género e inmigración (re)producidos en las políticas sociales y sus consecuencias para la acción e integración social", en: CACHÓN, L. y LAPARRA, M. (comps.). Inmigración y políticas sociales, Barcelona, Bellaterra, pp. 239-267.

ANDERSON, B. (2000): Doing the dirty work?: the global politics of domestic labour, London, Zed Books.

BADE, K. J. (2000): Europa en movimiento: las migraciones desde finales del siglo XVIII hasta nuestros días, Barcelona, Crítica.

BAUDER, H. (2005): "Habitus, rules of the labour market and employment strategies of immigrants in Vancouver, Canada", Social \& Cultural Geography, 6 (1), pp. 82-97.

BENERÍA, L. (2008): "The Crisis of Care, International Migration and Public Policy", Feminist Economics, 14 (3), pp. 1-21.

BOURDIEU, P. (1991): El sentido práctico, Madrid, Taurus.

BOURDIEU, P. (1997): Razones prácticas. Sobre la teoría de la acción, Barcelona, Anagrama.

BOURDIEU, P. (2003): La dominación masculina, Barcelona, Anagrama.

BRAH, A. (1987): "Diferencia, diversidad y diferenciación”. En HOOK, B. et. Al., Otras inapropiables, feminismo desde las fronteras, Madrid, Traficantes de sueños, pp.107-137.

CARRASCO, C. (2013): "El cuidado como eje vertebrador de una nueva economía”, $邓$ Cuadernos de Relaciones Laborales, 31 (1), pp. 39-56.

CATARINO, C. y OSO, L. (2000): "La inmigración femenina en Madrid y Lisboa. Hacia una etnización del servicio doméstico y de las empresas de limpieza", Papers Revista de sociología, 60, pp. 183-207.

COLECTIVO IOE (2001): Mujer, inmigración y trabajo, Madrid. IMSERSO-Ministerio de Trabajo y Asuntos Sociales.

DEPARTAMENT D’ESTADÍSTICA, Ajuntament de Barcelona. Disponible en http:// www.bcn.cat/estadistica/catala/dades/barris/tdemo/imi/taxes2012.htm [Consulta Marzo 2014].

ESCRIVÁ, A. (2000): “The position and status of migrant women in Spain”, en: ANTHIAS, F. y LAZARADIS, G. (eds.), Gender and Migration in Southern Europe. New York: Berg, pp. 199-226.

FERRERA, M. (1996): “The «Southern Model» of Welfare in Social Europe”, Journal of European Social Policy, 6/1 pp. 17-37.

GIL ARAÚJO, S. y GONZÁLEZ-FERNÁNDEZ, T. (2014): "International migration, public policies and domestic work Latin American migrant women in the Spanish domestic work sector". Women's Studies International Forum, 46, pp. 13-23.

GREGORIO, C. (1998): La migración femenina y su impacto en las relaciones de género, Madrid, Narcea.

GREGORIO, C. (2010): "Debates feministas en el análisis de la inmigración no comunitaria en el Estado español. Reflexiones desde la Etnografía y la Antropología Social", Relaciones Internacionales, 14. Disponible en: <http://www. relacionesinternacionales.info/ojs/index.php?journ

al=Relaciones_Internacionales\&page $=$ article \&op $=$ view\&path $\% 5 \mathrm{~B} \% 5 \mathrm{D}=217>$

[Consulta: Julio 2010] 
GUTIÉRREZ-RODRÍGUEZ, E. (2013): “Trabajo doméstico-trabajo afectivo: sobre heteronormatividad y la colonialidad del trabajo en el contexto de las políticas migratorias de la UE", Revista de Estudios Sociales, 45 (35), pp. 123-134.

HAMMERSLEY, M. y ATKINSON, P. (1994): Etnografía. Métodos de investigación, Barcelona, Paidos.

HINOJOSA GORDONAVA, A. (2009): Buscando la vida: Familias bolivianas transnacionales en España. CLACSO-Fundación PIEB: La Paz. Disponible en:

http://bibliotecavirtual.clacso.org.ar/ar/libros/coedicion/hinojosa/> [Consulta: Abril 2010]

LAHIRE, B. (2004): El hombre plural, Barcelona, Bellaterra.

LAPARRA, M. (ed.) (2003): Extranjeros en el purgatorio: integración social de los inmigrantes en el espacio local, Barcelona, Bellaterra.

LÓPEZ SALA, A. M. (2005): Inmigrantes y Estados: la respuesta política ante la cuestión migratoria, Barcelona, Anthropos.

MARTÍN PÉREZ A. (2009) Les étrangers en Espagne. La file d'attente devant les bureaux de l'immigration, París, L'Harmattan.

MARTÍN PÉREZ, A. (2010) "La práctica cotidiana de los derechos de ciudadanía en los Servicios Públicos de Bienestar". Miscelánea Comillas: Revista de Ciencias Humanas y Sociales 68 (133), pp. 609-638.

MARTÍNEZ DE LIZARRONDO, A. (2009): "Una mirada a los planes de integración de las comunidades autónomas" en Cachón, Lorenzo y Laparra, Miguel (eds.) (2009): Inmigración y políticas sociales, Barcelona, Bellaterra. pp. 51-74.

MORENO, L. (2002): "Bienestar mediterráneo y «supermujeres»", Revista Española de Sociología, 2, p. 41-57.

MOROKVASIC, M. (1984). "Birds of passage are also women”, International Migration Review, 18(4), pp. 886-907.

PARELLA RUBIO, S. (2003): Mujer, inmigrante y trabajadora: la triple discriminación, Rubí, Anthropos.

PARELLA RUBIO, S. (2012): "Familia transnacional y redefinición de los roles de género. El caso de la migración boliviana en España”, Papers: revista de sociología, 97, pp. 661-684.

PEDONE, C. (2007): "Familias transnacionales ecuatorianas: estrategias productivas y reproductivas", en: BRETÓN, V. et al. (eds.). Ciudadanía y Exclusión: Ecuador y España frente al espejo, Madrid, Los Libros de la Catarata, pp. 251-278.

PEDONE, C. (2014): "Rupturas y continuidades de los roles de género en contextos migratorios transnacionales: Relatos sobre sexualidad y salud reproductiva de los hijos e hijas de la inmigración ecuatoriana en Cataluña”. Papeles del CEIC, International Journal on Collective Identity Research, 2, (6)-111. Disponible en: http://dx.doi.org/10.1387/pceic.12968 [Consulta: Diciembre 2014]

PLA DE CIUTADANIA I IMMIGRACIÓ 2005-2008 (2005), Generalitat de Catalunya, Departament d'immigració, Disponible en: www20.gencat. cat/docs/dasc/01Departament/08 Publicacions /Ambits\%20tematics/ Immigracio/03 publiforacoleccio/Anteriors/08placiutadania immigracio 0508/2006placiutadania05_0.pdf [Consulta: Mayo 2008].

PLA DE CIUTADANIA I IMMIGRACIÓ 2009-2012 (2009), Generalitat de Catalunya, Departament d'immigració, Disponible en: www20.gencat.cat/docs/dasc/01 Departament/08 Publicacions/Ambits \%20tematics/Immigracio/03publiforacoleccio. pdf [Consulta: Febrero 2010] 
PLAN ESTRATÉGICO DE CIUDADANÍA E INTEGRACIÓN 2007-2010 (2007), Ministerio de Trabajo y Asuntos Sociales, Secretaría de Estado de Inmigración y Emigración, Disponible en: http://extranjeros.empleo.gob.es/es/IntegracionRetorno/ Plan_estrategico/pdf/PECIDEF180407.pdf [Consulta: Julio de 2008]

PHIZACKLEA, A. (1994): "A single or segregated market", en AFSHAR, H. y MAYNARD, M. (Eds.), The Dynamics of "Race" and Gender. Some Feminist Interventions, London, Taylor \& Francis, pp. 172-181.

RIBAS, N. et al. (2000): "Políticas de formación para mujeres inmigrantes. El caso de la ciudad de Barcelona”, Papers Revista de sociología, 60, pp. 365-379.

SANTAMARÍA, E. (2002): La incógnita del extraño. Una aproximación a la significación sociológica de la 'inmigración no comunitaria', Barcelona, Anthropos.

SASSEN, S. (2003): Contrageografías de la globalización: género y ciudadanía en los circuitos transfronterizos. Madrid, Traficantes de Sueños.

SAYAD, A. (2010): La doble ausencia. De las ilusiones del emigrado a los padecimientos del inmigrado. Barcelona, Anthropos.

SUÂREZ NAVAZ, L. (2007): "Familias en movimiento. El caso de las mujeres rumanas en España", Migraciones, 21, pp. 235-257.

VEGA SOLÍS, C. (2009): Culturas del cuidado en transición. Espacios, sujetos e imaginarios en una sociedad de migración, Barcelona, UOC.

ZAPATA, R. (2010): “Teorizando el enfoque español: coherencia de políticas públicas, interculturalidad y gobernanza multinivel" en AJA, E. et al., Inmigración y crisis económica. Impactos actuales y perspectivas de futuro. Anuario de la inmigración en España 2010, Cidob, Bellaterra, pp. 276-293.. 